\title{
ORNAMENTAÇÃO DO TIPO PADRÃO-CROTON EM GRÃOS DE PÓLEN DE SCROPHULARIACEAE DO BRASIL
}

\author{
Francisco de Assis Ribeiro dos SANTOS e Therezinha Sant'Anna MELHEM
}

\begin{abstract}
RESUMO. Ornamentação do tipo padrão-Croton em grãos de pólen de Scrophulariaceae do Brasil. Foram estudados, sob microscopia óptica e eletrônica de varredura, grãos de pólen acetolisados de algumas espécies brasileira dos gêneros Agalinis, Esterhazya, Melasma e Physocalyx (Scrophulariaceae). As análises revelaram que algumas espécies têm grãos de pólen com superfície pilada, estando os pilos organizados num padrão reticulado (retipilado) ou no conhecido padrão-Croton, que é caracterísitico de algumas famílias (Buxaceae. Euphorbiaceae e Thymelaeaceae). Os pilos das espécies estudadas se caracterizaram pelo reduzido tamanho, de modo que a ornamentação em microscopia óptica é definida como microrreticulado, estando os pilos (alguns multicapitados) visíveis individualmente apenas sob microscopia eletrônica de varredura. A literatura palinológica não tem referência de padrão-Croton em espécies de Scrophulariaceae.
\end{abstract}

Palavras-chave. Scrophulariaceae, padrão-Croton, grãos de pólen, Agalinis, Esterhazya, Melasma, Physocalyx.

RESUMEN. Ornamentación de tipo patrón-Croton en granos de polen de Scrophulariaceae de Brasil. En este trabajo, granos de polen acetolisados de algunas especies brasileñas de los géneros Agalinis, Esterhazya, Melasma y Physocalyx (Scrophulariaceae) fueron estudiados, en microscopia de luz y eletrónica de barrido (MEB). Los análisis mostraron que algunas especies tienen granos de polen pilados, estando los pilos ordenados en retículo (retipilados) o en el conocido patrón-Croton, que es típico de las familias Buxaceae, Euphorbiaceae y Thymelaeaceae. Los pilos de las especies estudiadas fueron caracterizados por su talla reducida, de modo que la ornamentación es definida en microscopia de luz como microreticulada, siendo los pilos (algunos multicapitados) visibles solamente en MEB. No hay datos del patrón-Croton en las especies de Scrophulariaceae en la literatura palinológica.

Palabras clave. Scrophulariaceae, patrón-Croton, granos de polen, Agalinis, Esterhazya, Melasma, Physocalyx.

ABSTRACT. Ornamentation Croton-pattern in the pollen grains of Brazilian Scrophulariaceae. This study dealt with acetolysed pollen grains of some Brazilian species of the genera Agalinis, Esterhazya, Melasma and Physocalyx (Scrophulariaceae). They were studied, using light microscopy and SEM. The analyses, showed that some species have pilate pollen grains, the pila being organized in a reticulum (retipilate) or in the known Croton-pattern that is common in the families Buxaceae, Euphorbiaceae and Thymelaeaceae. The pila of the species studied were notable for their reduced size, so that the ornamentation under light microscopy is seen as microreticulate, the pila (some multicapitate) being visible only under SEM. There are no reports of the Croton-pattern in species of Scrophulariaceae in the palynological literature.

Key words. Scrophulariaceae, Croton-pattern, pollen grains, Agalinis, Esterhazya, Melasma, Physocalyx. 


\section{INTRODUÇÃO}

Erdtman (1952), para grãos de pólen de espécies de Buxaceae, Euphorbiaceae e Thymelaeaceae, descreveu um tipo especial de ornamentação de exina que denominou padrãoCroton, uma referência ao gênero Croton (Euphorbiaceae), e definiu como crotonóide uma variação deste tipo de escultura.

Posteriormente, o padrão-Croton ou crotonóide tem sido referido, direta ou indiretamente, para os seguintes grupos taxonômicos: Callitrichaceae (Martinsson, 1993), Euphorbiaceae (Salgado-Labouriau, 1973), Icacinaceae (Lobreau-Callen, 1972; Lobreau-Callen \& Srivastava, 1974) e Thymelaeaceae (Barth \& Silva, 1965; Rosello \& Melhem, 1998).

Para a família Scrophulariaceae, ornamentação similar ao tipo padrão-Croton pode ser observada na ilustração dos grãos de pólen de Odontiella tenuifolia (Bolliger \& Wick, 1990, cf. fig. 33), onde os autores fazem referência ao conceito de Erdtman (1952) para este tipo de ornamentação, mas definiram a exina como retipilada. Rosello \& Melhem (1998) relacionaram outros trabalhos onde as ilustrações dos grãos de pólen mostram a exina com a aparência do padrão-Croton ou crotonóide e foram descritos com outras denominações.

Santos (1997), ao estudar os grãos de pólen de 84 espécies de Scrophulariaceae que ocorrem no Brasil, encontrou para algumas espécies dos gêneros Agalinis Benth., Esterhazya J.C. Mikan, Melasma Berg. e Physocalyx Pohl (Tribo Buchnereae Subfamília Rhinanthoideae) pólen com a ornamentação semelhante ao padrão-Croton. Os gêneros Esterhazya e Physocalyx são endêmicos do Brasil. Para outros gêneros da tribo Buchnereae, Buchnera L., Escobedia Ruiz \& Pávon, Schizosepala G.M. Barroso e Velloziella Baill., os grãos de pólen das espécies estudadas não mostraram ornamentação similar ao padrão-Croton e foram descritos por Santos (1997) como microrreticulados ou psiladoperfurados, sob microscopia eletrônica de varredura.. Além dos dados de Santos (1997), a literatura palinológica não registra nenhuma citação para espécies dos gêneros Melasma e Physocalyx, havendo entretanto referências para Agalinis (Jones et al. 1995) e Esterhazya (Minkin \& Eshbaugh, 1989).

O presente trabalho visa descrever os grãos de pólen de espécies brasileiras com ornamentação do tipo padrão-Croton, com base em microscopia óptica (MO) e microscopia eletrônica de varredura (MEV).

\section{MATERIAL E MÉTODOS}

Foram analisados os grãos de pólen de sete espécies pertencentes a Agalinis, Esterhazya, Melasma e Physocalyx (tabela 1), das quais três são espécies novas que se encontram em fase de publicação (conforme Souza, 1996).

Para cada espécie, mais de um espécime de foram analisados, entretanto a título de referência, apenas um, considerado como espécime padrão, foi utilizado para caracterizar e ilustrar palinologicamente a respectiva espécie, é citado na tabela 1 , outros foram utilizados como espécimes de comparação para melhor caracterizar cada táxon. No Apêndice consta a lista de todos os espécimes analisados.

Para análise sob microscopia óptica, adotou-se o método de acetólise de Erdtman (1960), sendo que antes de iniciar a técnica o material polinífero (flores e/ou botões florais) ficou em ácido acético glacial por um período mínimo de 24 horas. Tomou-se, sempre que possível, anteras de mais de uma flor por espécime para estudo, a fim de uniformizar a amostra (Salgado-Labouriau et al., 1965).

Nos grãos de pólen do espécime-padrão foram tomadas, ao acaso, 25 medidas dos diâmetros polar e equatorial em vista equatorial 

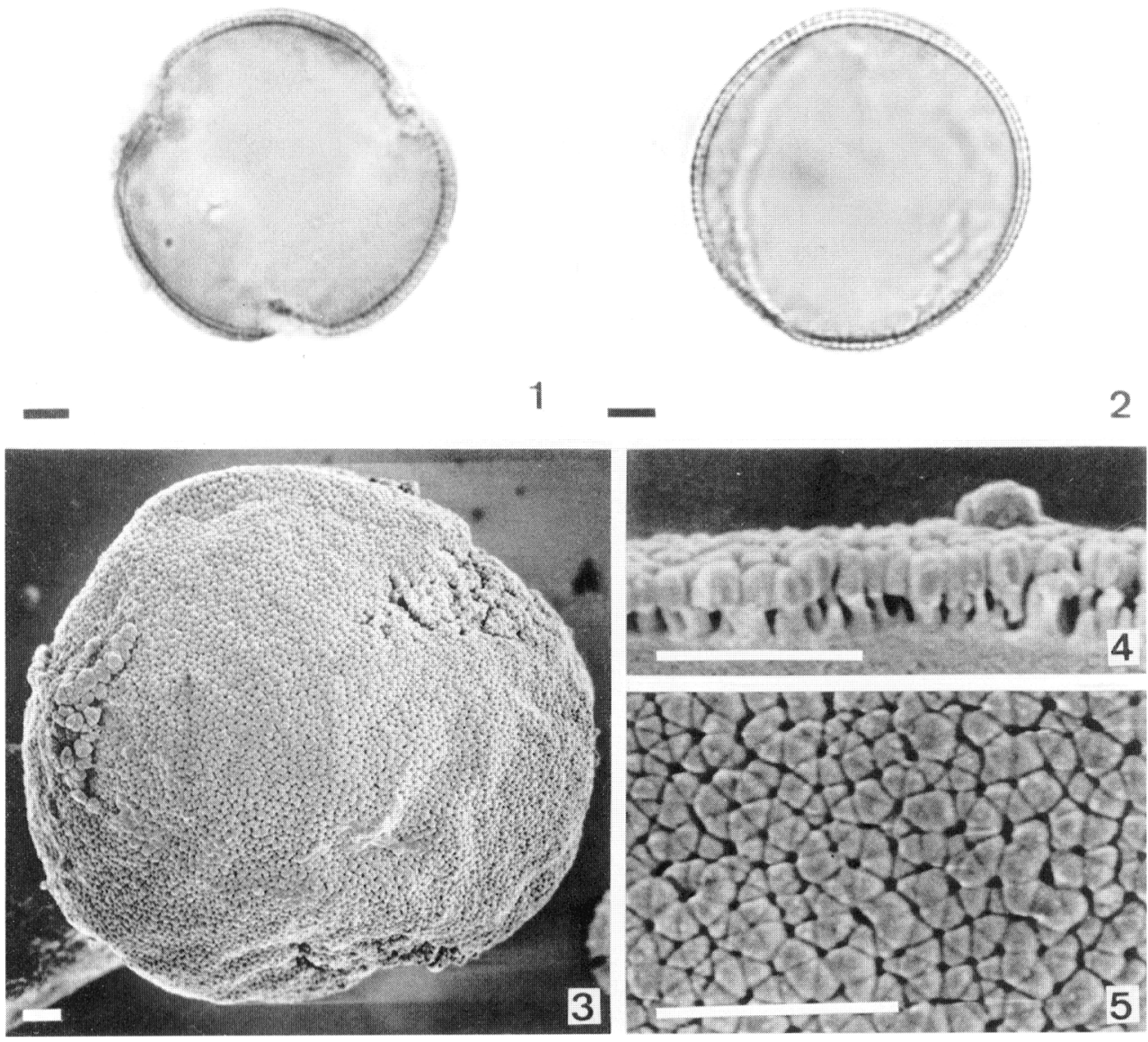

Figuras 1-5. Grãos de pólen de Agalinis communis (Cham. \& Schltdl.) D’Arcy: 1-2 - corte óptico das vistas polar e equatorial, respect.; 3 - vista polar (MEV); 4 - estrutura da exina (MEV); 5 - detalhe da superfície da exina $(\mathrm{MEV})$. Escalas $=2,5 \mu \mathrm{m}$

e 10 medidas da espessura da exina; calculouse a média aritmética para caracterizar a classe de tamanho do pólen, visando apenas complementar sua descrição.

Para as análises sob microscopia eletrônica de varredura, após a acetólise os grãos de pólen foram desidratados em série alcoólica crescente, montados nos portaespécimes, metalizados e fotomicrografados em microscópios Zeiss DSM940 e Jeol JSM6300. Para análise da estrutura da exina, sob microscopia eletrônica de varredura, adotou-se o método de crio-seccionamento dos grãos de pólen (Muller, 1973).

\section{RESULTADOS}

Morfologia geral - Os grãos de pólen das espécies estudadas são de tamanho médio (entre 26 e $50 \mu \mathrm{m}$ ); isopolares; com forma variando de suboblata (Melasma rhinanthoides) a 


\begin{tabular}{|c|c|c|c|c|c|c|c|}
\hline Espécies & Voucher & Diâmetro polar a & Diâmetro equatorial ${ }^{a}$ & $\mathrm{P} / \mathrm{E}$ & Exina & ${ }^{b}$ Sexina ${ }^{a}$ & Nexina $^{b}$ \\
\hline Agalinis communis & N. Moura s.n. (SP5353) & $44(40-50)$ & $45(42-49)$ & 0,98 & 2,4 & 1,3 & 1,1 \\
\hline Agalinis itambensis & CFCR 3062 & $39^{b}$ & $41^{b}$ & 0,95 & 2,2 & 1,4 & 0,8 \\
\hline Esterhazya splendida & M.L. Guedes 687 & $35(34-38)$ & $38(35-39)$ & 0,92 & 2,3 & 1,3 & 1,0 \\
\hline Esterhazya nanuzae & CFCR 10596 & $36(33-38)$ & $39(37-41)$ & 0,92 & 2,2 & 1,5 & 0,8 \\
\hline Melasma rhinanthoides & A. Bidá 391 et al. & $27^{b}$ & $33 \mathrm{~b}$ & 0,82 & 1,9 & 1,1 & 0,8 \\
\hline Physocalyx scaberrimus & CFSC 1524 & $34(30-38)$ & $34(32-37)$ & 1,00 & 1,9 & 1,1 & 0,8 \\
\hline Physocalyx coriaceus & R.M. Harley 15516 & $36^{\mathrm{b}}$ & $37^{b}$ & 0,97 & 1,8 & 1,2 & 0,6 \\
\hline
\end{tabular}

${ }^{a}$ Média (faixa de variação), $\mathrm{n}=25$, valores em mm. ${ }^{b}$ Média, $\mathrm{n}<25$, valores em $\mu \mathrm{m}$.

Tabela 1. Dados morfométricos dos grãos de pólen e do material botânico estudado.

esférica (Physocalyx scaberrimus), sendo oblato-esferoidal nas demais espécies estudadas; amb circular; 3(4)-colpoidados, com membrana apertural fortemente ornamentada $\mathrm{e}$ frágil; exina microrreticulada sob microscopia óptica e, sob microscopia eletrônica de varredura, com um padrão de ornamentação bastante incomum entre as espécies da família, do tipo do padrão-Croton.

No que se refere ao número de colpóides, Physocalyx coriaceus apresentou a maior variação observada, com grãos de pólen 3colpoidados (80\%) e 4-colpoidados (20\%) e $P$. scaberrimus mostrou raros grãos de pólen anormais 6-pantocolpoidados, em geral, maiores que os 3-colpoidados.

\section{Descrição da exina}

Agalinis - Espécies estudadas: A. communis (Cham. \& Schltdl.) D'Arcy (fig. 1-5) e Agalinis itambensis V. C. Souza \& S. I. Elias (fig. 612). Nos grãos de pólen destas espécies a espessura da exina variou entre 2,2 e $2,4 \mu \mathrm{m}$, sexina mais espessa do que a nexina (tabela 1), afinando-se em direção dos colpóides (fig. 7). Sob microscopia óptica, a superfície da exina é microrreticulada (fig. 6-8), muros simplescolumelados; em corte óptico a exina mostra uma estrutura pilada. Sob MEV, em A. communis, em menor aumento, a exina mostrase pilada (fig. 3), com muitos pilos multicaptados, detalhado em maior aumento na figura 4 , cujas cabeças, mais altas do que largas, têm secção transversal triangular, estando organizados no padrão-Croton (fig. 5); em Agalinis itambensis, em menor aumento, a exina é microrreticulada (fig. 9); em maior aumento (fig. 10) nota-se, ao redor da extremidade da abertura, que os muros dos microrretículos têm uma organização semelhante às "pétalas de uma flor", podendose observar a presença de pilos multicaptados (fig. 11), cujas cabeças (6-7) organizam-se em anel, formando uma imagem próxima a do padrão-Croton em vista frontal (fig. 12). As figuras 11 e 12 mostram ainda que neste táxon a vista superficial não é tão homogênea como em A. communis (fig. 5), pois há áreas onde os pilos estão isolados ou constituindo microrretículos bastante heterogêneos.

Esterhazya - Espécies estudadas: E. splendida Mikan (fig. 13-19) e Esterhazya nanuzae V.C. Souza (fig. 20-21). A espessura da exina nas espécies analisadas deste gênero variou de 2,2 a 2,4 $\mathrm{mm}$ de espessura, sexina mais espessa do que a nexina (tabela 1), afinando-se em direção dos colpóides (fig. 13 e 20). Em E. splendida observou-se, sob MEV, num grão de pólen seccionado, que a nexina na sua face interna não é lisa e que na região da membrana apertural apresenta-se rachada (fig. 17), o que torna esta área do colpóide muito frágil (fig. 16). Sob microscopia óptica, a análise L.O. revelou uma 

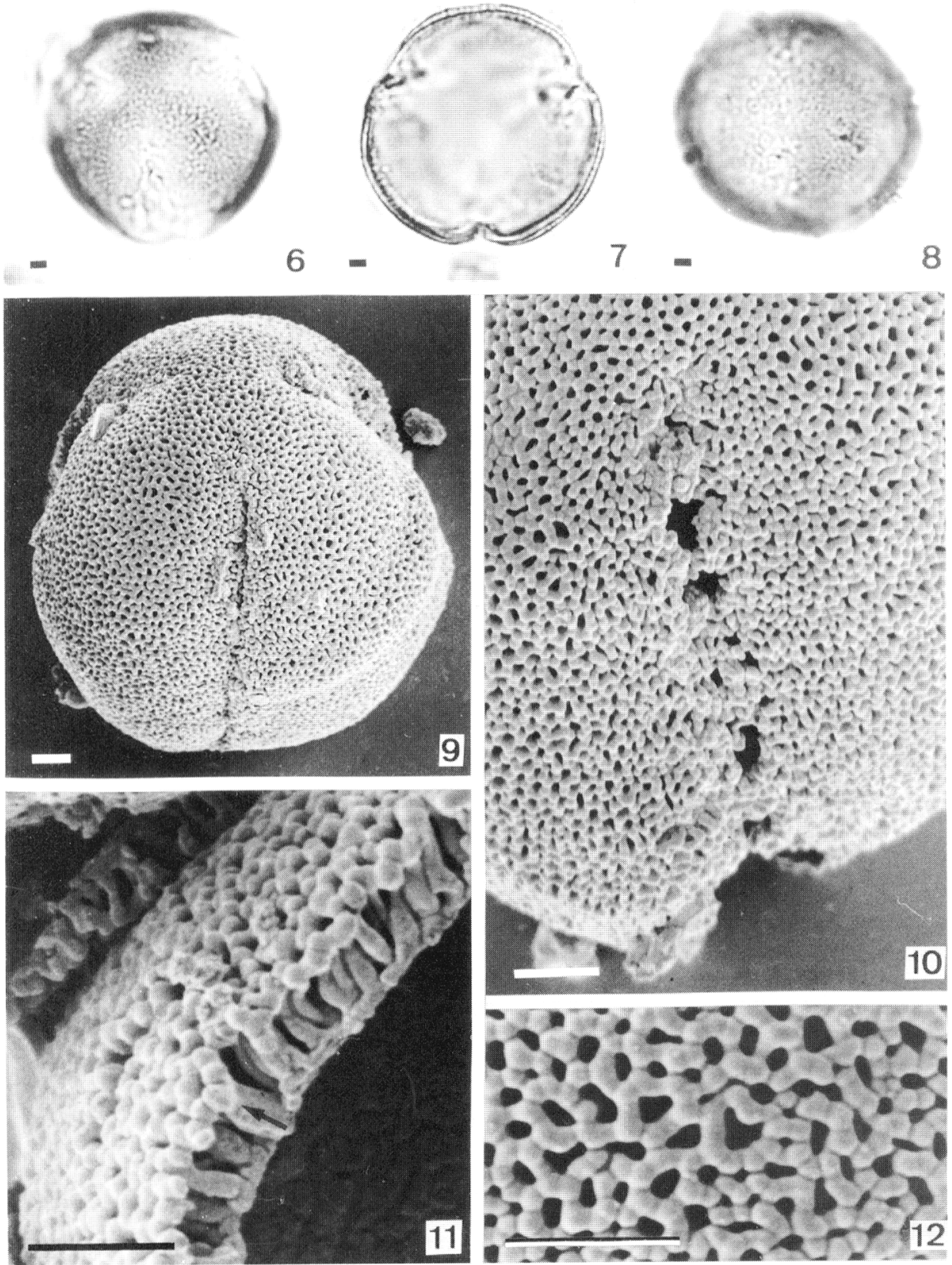

Figuras 6-12. Grãos de pólen de Agalinis itambensis V.C. Souza \& S.I. Elias: 6 - superfície, vista polar; 7 - corte óptico, vista polar; 8 - superfície, vista equatorial; 9 - superfície (MEV); 10 - detalhe da região apertural (MEV); 11 - estrutura e ornamentação da exina (MEV), seta = pilo multicapitado; 12 - detalhe da superfície $(\mathrm{MEV})$. Escalas $=2,5 \mu \mathrm{m}$. 
exina microrreticulada (fig. 15). Mesmo sob $\mathrm{MEV}$, em baixos aumentos, a ornamentação ainda é visualizada como microrreticulada (fig. 20); na figura 16 , na região equatorial do mesocolpo, próximo ao colpóide, nota-se uma área onde o padrão-Croton pode ser identificado. Em grão de pólen seccionado (fig. 19, seta), nota-se a ocorrência de pilos multicapitados. Outro caráter importante observado sob MEV, foi o posicionamento dos pilos multicapitados, num arranjo reticulóide de modo que suas cabeças exibem o tipo escultural denominado de padrão-Croton (fig. 18). Os pilos têm cabeças triangulares em vista superficial (fig. 18 e 21).

Melasma - Espécie estudada: M. rhinanthoides (Cham.) Benth. (fig. 22-26). Exina com 1,9 $\mu \mathrm{m}$ de espessura; sexina mais espessa do que a nexina (tabela 1) e, em geral, se afina nas proximidades das aberturas (fig. 22). A superfície da exina apresentou-se microrreticulada sob MO (fig. 26). Em geral, sob MEV, a superfície da exina é visualizada, em pequenos aumentos, como microrreticulada (fig. 23). Em grandes aumentos, em secção transversal, observou-se que a exina tem uma estrutura pilada, que os pilos são constituídos por um colo grosso (columela) que sustenta uma cabeça alongada provida de cúspide (fig. 24); os pilos de diferentes tamanhos ficam distribuídos num padrão reticulóide de modo que alguns estão reunidos em grupos (5-6) formando rosetas como no padrão-Croton, mas na maioria das vezes eles estão isolados ou unidos em grupos de 2-3 (fig. 25, seta). A visualização do padrão-Croton fica dificultada pelas diferenças no tamanho dos pilos que se organizam em roseta (fig. 25).

Physocalyx - Espécies estudadas: $P$. scaberrimus Philcox (fig. 27-30) e Physocalyx coriaceus V. C. Souza (fig. 31-35). Neste gênero a exina é relativamente tênue, alcançando a espessura média máxima de 1,9 $\mathrm{mm}$, sexina mais espessa do que a nexina (tabela 1); a sexina se estreita em direção às aberturas (fig. 33). Sob MO, a análise de L.O. revelou no primeiro nível de focalização pontos claros esparsos, irregularmente distribuídos (pilos) e um microrretículo (fig. 31-32), às vezes de muros interrompidos. Sob MEV, os pilos estão organizados em rosetas, semelhantes ao padrãoCroton (fig. 29 e 35). O tamanho reduzido dos pilos faz com que em pequenos aumentos, sob MEV, a exina seja observada como microrreticulada (fig. 28 e 34). O grão de pólen de $P$. scaberrimus seccionado exibiu exina com pilos com cabeças conspícuas e arredondadas, colos (columelas) finos e nexina espessa, internamente ornamentada (fig. 30).

\section{DISCUSSÃO}

Erdtman (1952) ao definir o padrãoCroton, caracterizou esta estrutura pela presença de pilos, freqüentemente com secção transversal triangular, distribuídos num padrão reticular ao redor de fovéolas de modo que cada pilo margeia três fovéolas; e cada fovéola é limitada por 5-6 pilos, raras vezes por um número maior.

Para Punt et al. (1994) o padrão-Croton representa um tipo de ornamentação caracterizada pela presença de anéis com cinco ou seis (às vezes mais) elementos de sexina (pilos), proeminentes, geralmente triangulares e distribuídos ao redor de uma área circular.

Rosello \& Melhem (1998) fizeram um estudo minucioso dos grãos de pólen de 21 espécies brasileiras de Thymelaeaceae, ao nível de microscopia óptica, eletrônica de varredura e de transmissão. Estas autoras mostraram, em todos os táxons, que a análise de LO permite identificar quatro níveis de focalização da exina, bem distintos entre si. As imagens obtidas nas análises de LO e nas elétronmicrografias revelaram que sobre a camada basal a sexina tem uma estrutura columelada; as columelas sustentam muros (às vezes com uma estrutura não muito organizada) em forma 

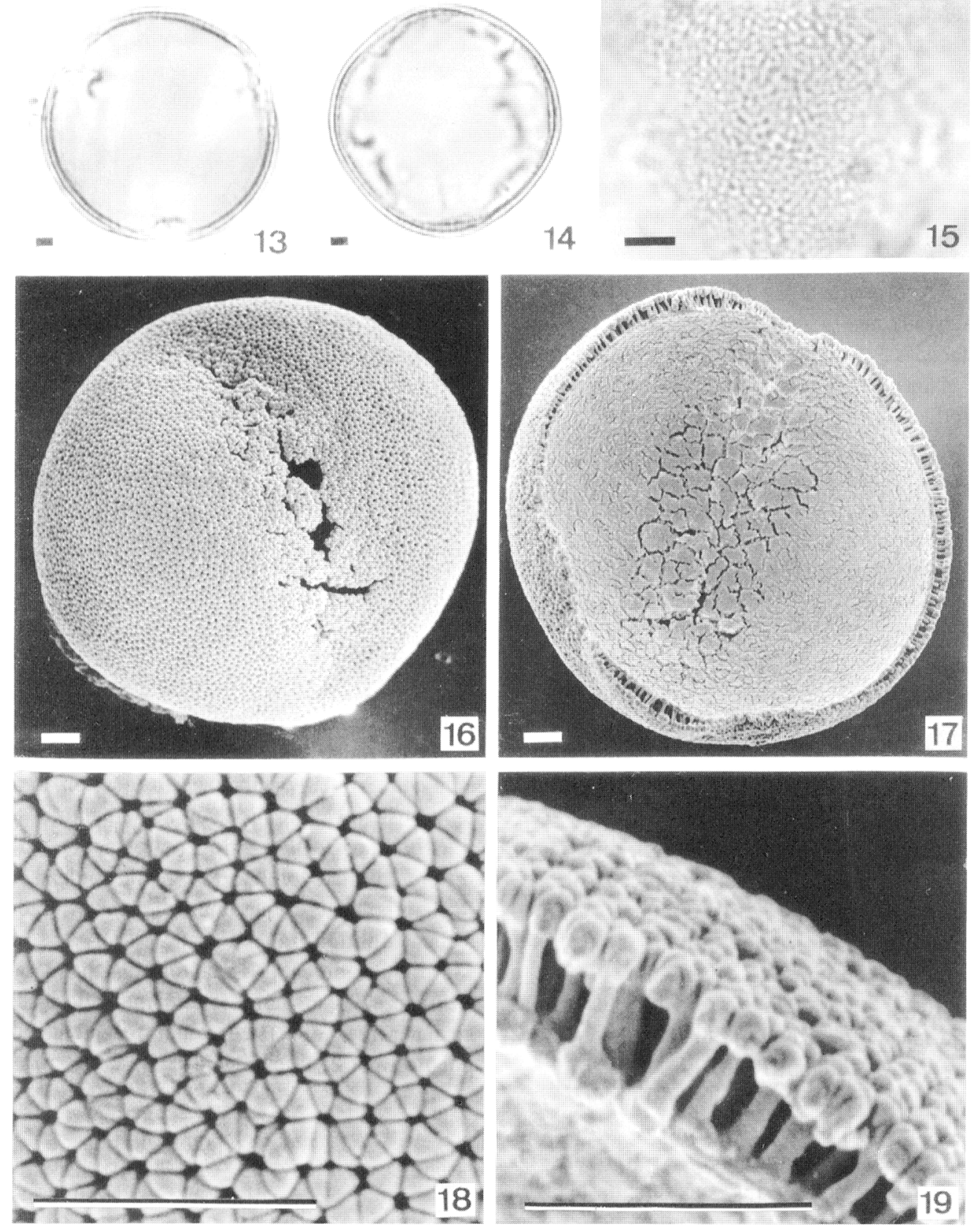

Figuras 13-19. Grãos de pólen de Esterhazya splendida Mikan: 13-14 - corte óptico das vistas polar e equatorial, respect.; 15 - detalhe da superfície; 16 - grão de pólen inclinado, vista equatorial (MEV); 17 grão de pólen seccionado longitudinalmente, superfície interna (MEV); 18 - detalhe da superfície (MEV); 19 - detalhe da estrutura da exina (MEV). Escalas $=2,5 \mu \mathrm{m}$ 
de anel sobre os quais ficam 6-7 (raramente 5) unidades de ornamentação, aproximadamente ou nitidamente tetraédricas, com ápice espiculado. No padrão crotonóide as unidades de ornamentação não são nitidamente individualizadas em rosetas como no padrãoCroton, que segundo Salgado-Labouriau (1973), têm uma distribuição radial como "pétalas de uma flor", sobre uma camada suporte de sexina.

A ornamentação do tipo padrão-Croton observada nos grãos de pólen das espécies aqui estudadas é diferente da citada pela literatura para os táxons cujos grãos de pólen são classicamente referidos com este tipo de ornamentação.

Nas espécies de Scrophulariaceae aqui estudadas, a ornamentação do tipo padrãoCroton só pode ser assim definida sob MEV e caracterizada pela presença de pilos muito pequenos arranjados em rosetas ou de pilos multicapitados (Agalinis communis, Agalinis itambensis, Esterhazya splendida e Esterhazya nanuzae) cujas cabeças é que se organizam em rosetas, sem a presença de um semiteto para sustentar estes elementos uma vez que a própria columela (tubular, algumas vezes) os sustenta. Em outras famílias (como Buxaceae, Callitrichaceae, Euphorbiaceae e Thymelaeaceae), o padrão-Croton diferenciase primeiramente por sua definição sob MO devido ao tamanho dos elementos que o compõem, e pelo posicionamento desses elementos circulares a triangulares sobre 0 semiteto, e também arranjados em rosetas (Erdtman, 1952; Khan, 1968; Martinsson, 1993; Rosello \& Melhem, 1998).

Minkin \& Eshbaugh (1989) descreveram os grãos de pólen de Esterhazya splendida, sob MO, como sendo 3-colpados e retipilados. Os grãos de pólen dos vários espécimes deste táxon aqui examinados exibiram colpóides (cf. fig. 16 e 17) e exina microrreticulada, sob MO (fig.15), e com uma ornamentação do tipo padrão-Croton, sob MEV (fig. 18).

Nos grãos de pólen das espécies aqui estudadas, Agalinis communis, Esterhazya splendida, Esterhazya nanuzae, Physocalyx coriaceus e Physocalyx scaberrimus, a ornamentação tipo padrão-Croton é conspícua quando a exina é observada sob MEV, em grandes aumentos. Nos grãos de pólen Agalinis itambensis e Melasma rhinanthoides, as rosetas na superfície da exina são menos conspícuas, mesmo sob MEV, em grandes aumentos.

As semelhanças observadas na ornamentação em rosetas na superfície da exina nas Scrophulariaceae aqui estudadas (fig. $5 \mathrm{e}$ 18) com a encontrada nas Euphorbiaceae e Thymelaeaeceae onde ocorre o padrão-Croton, e considerando-se que a estrutura dessas rosetas é distinta, conforme já comentado, optou-se pelo termo "tipo padrão-Croton", ou seja, parecido com o padrão-Croton; desaconselhase o termo retipilado, pois neste caso os pilos se organizam segundo um retículo, sem a típica aparência de roseta.

Santos (1997), observando a tendência evolutiva dos táxons estudados por Souza (1996) e os dados palinológicos disponíveis na literatura, sugeriu que, nas Scrophulariaceae como um todo, tenha havido a redução do teto com a individualização dos pilos que tomaram uma posição reticulóide, ou semelhante à do tipo padrão-Croton. Filogeneticamente, isto reflete a linha evolutiva Scrophularioideae $\rightarrow$ Rhinanthoideae; o que também é corroborado pela tendência plantas autótrofas (Scrophularioideae) $\rightarrow$ plantas hemi-parasitas (Rhinanthoideae), conforme foi indicado desde cedo por Boeshore (1920) e, mais recentemente, por Bolliger \& Wick (1990).

\section{APÊNDICE}

\section{Espécimes analisados}

A. communis (Cham. \& Schltdl.) D’Arcy - Paraná: Curitiba, R. Kummorow 2873 e J. Cordeiro, 4.II.1987, det. V.C. Souza (HRB); Palmeiras, 

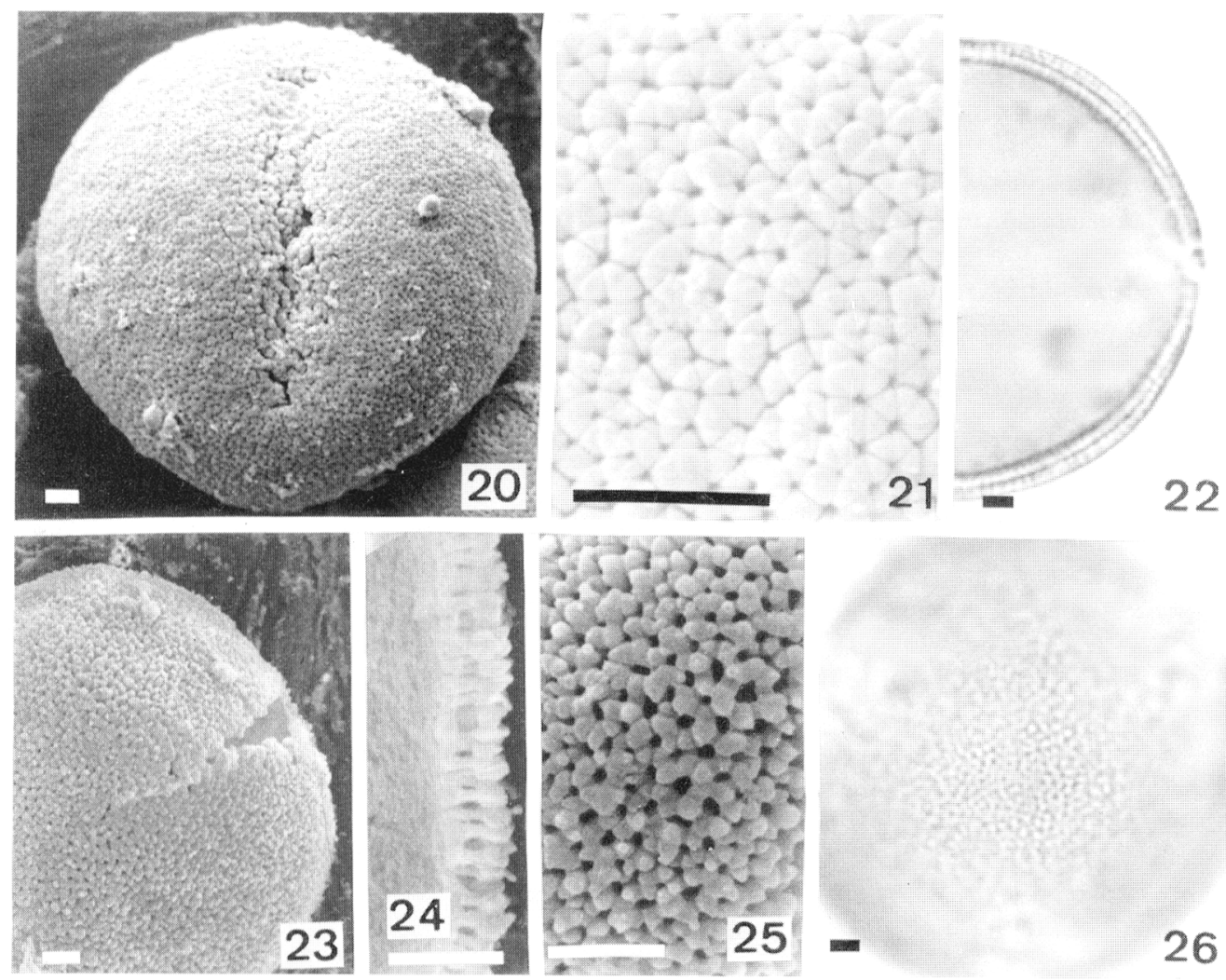

Figuras 20-26. Grãos de pólen de espécies de Esterhazya e de Melasma. E. nanuzae V. C. Souza: 20 superfície, vista equatorial (MEV); 21 - detalhe da superfície (MEV). M. rhinanthoides (Cham.) Benth. 22 - corte óptico da exina; 23 - superfície, parte da vista polar (MEV); 24 - detalhe da estrutura da exina $(\mathrm{MEV}) ; 25$ - detalhe da superfície da exina (MEV); 26 - superfície, vista polar. Escala $=2,5 \mu \mathrm{m}$.

G. Hatschbach 7753, 20.II.1961, det. G. Hatschbach (MBM); Piraquara, G. Hatschbach 1171 , 1.II.1949, det. G. M. Barroso (MBM); São José dos Pinhaes, G. Hatschbach 42778, 4.III.1980, det. G. Hatschbach (MBM). São Paulo: São Paulo, N. Moura s.n., 14.III.1921, det. V.C. Souza (SP 5353*).

Agalinis itambensis V.C. Souza \& S.I Elias - Minas Gerais: Santo Antônio do Itambé, A. Furlan et al. s.n., CFCR 3062, 5.IV.1982, det. V.C. Souza (SPF*).

Esterhazya splendida J.C. Mikan - Bahia: Ferro Doido, G.M. Barroso s.n., 17.V.1975, det. G.M. Barroso (ALCB 20534); Serra do Pai Inácio, M.L. Guedes 687, 29.VI.1983, det. M.L. Guedes $\left(\mathrm{ALCB}^{*}\right)$. Mato Grosso: Posto Vale do Guaporé,
Dilson L. Amaral 55, 16.IV.1978, det. H.P. Bautista (HRB). Minas Gerais: Carangola, L.S. Leoni 1531, 24.IV.1991, det. J.R. Pirani (PABST). Paraná: Ipiranga, G. Hatschbach 25929, 21.XII.1970, det. G. Hatschbach (MBM). Esterhazya nanuzae V. C. Souza - Minas Gerais: Diamantina, N.L. Menezes et al. s.n., CFCR 11849, 30.III.1988, det. V.C. Souza (SPF); A.M. Giulietti 948-80, CFCR 36, 3.IV.1980, det. V.C. Souza (SPF); D.C. Zappi et al. s.n., CFCR 10596, 17.IV.1987, det. V.C. Souza (SPF*); Gouveia, N.L. Menezes et al. s.n., CFCR 11842, 29.II.1988, det. V.C. Souza (SPF); J. Semir et al. s.n., CFCR 9575, 24.II.1988, det. V.C. Souza (SPF).

Melasma rhinanthoides (Cham.) Benth. - Paraná: 

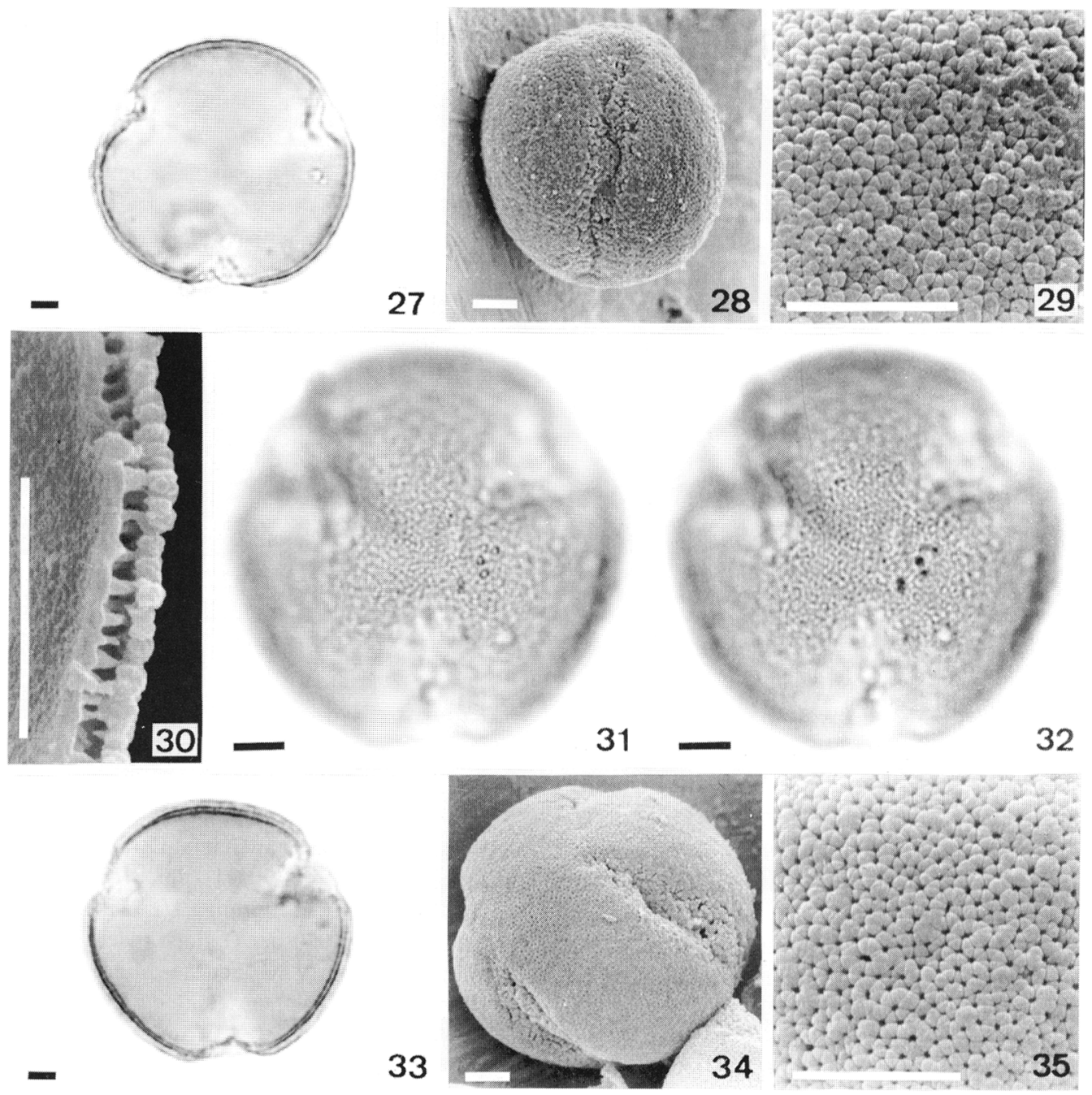

Figuras 27-35. Grãos de pólen de espécies de Physocalyx. P. scaberrimus Philcox (Fig. 27-30): 27 - corte óptico, vista polar; 28 - vista equatorial (MEV); 29 - detalhe da superfície (MEV); 30 - detalhe da estrutura da exina (MEV). P. coriaceus V.C. Souza (Fig. 31-35): 31-32 - vista polar, análise de LO; 33 - vista polar, corte óptico; 34 - superfície (MEV); 35 - detalhe da superfície (MEV). Escalas $=5 \mu \mathrm{m}$. 
Colombo, A. Bidá 391 et al., 14.III.1984, det. L.A. Acra (UPCB*); São Mateus do Sul, $G$. Hatschbach 13768, 8.II.1966, det. G. Hatschbach (MBM); Palmeira, J.M. Silva 1077, 28.II.1992, det. G. Hatschbach (HUEFS); Rio Azul, G. Hatschbach 29194, 24.II.1972, det. G. Hatschbach (MBM). São Paulo: São Paulo, W. Hoehne s.n., 10.III.1942, det. W. Hoehne (SPF 13860); W. Hoehne s.n., 14.II.1942, det. W. Hoehne (SPF 13861).

Physocalyx coriaceus V. C. Souza - Bahia: Barra da Estiva, R.M. Harley 15516, 27.I.1976, det. D. Philcox (CEPEC*); Rio de Contas, S.A. Mori e F. Benton 13523, 21.III.1980, det. A.M. Carvalho (CEPEC).

P. scaberrimus Philcox - Bahia: Água Quente, R.M. Harley 27524, 16.XII.1988, det. R.M. Harley (ESA); Mucugê, A.M. Giulietti et al. s.n., CFSC 1524, 20.VII.1981, det. V.C. Souza (SPF*); Piatã, R.M. Harley 24275, 15.II.1987, det. V.C. Souza (SPF).

AGRADECIMENTOS. Ao Dr. S. Nilsson (Swedish Museum of Natural History) por ter disponibilizado as facilidades do Laboratório de Palinologia; ao CNPq e à CAPES pela concessão de bolsa a T. S. Melhem e F.A.R. Santos, respectivamente; e ao Departamento de Ciências Biológicas da Universidade Estadual de Feira de Santana pela liberação de F.A.R. Santos para realizar este trabalho.

\section{REFERÊNCIAS BIBLIOGRÁFICAS}

ARGUE, C.L. -1993- Pollen morphology in the Selagineae, Manuleae (Scrophulariaceaae), and selected Globulariaceae, and its taxonomic significance. Amer. J. Bot. 80(6): 723-733.

BARTH, M.O. \& S.A.F. SILVA -1965- Catálogo sistemático dos pólens das plantas arbóreas do Brasil Meridional VII. Thymelaeaceae, Lythraceae, Lecythidaceae, Rhyzophoraceae e Combretaceae. Mem. Inst. Oswaldo Cruz Rio J. 63: 255-279

BOESHORE, I. -1920- The morphological continuity of Scrophulariaceae and Orobanchaceae. Contr. Bot. Lab. Morris Arbor. Univ. Penn. 5:139-177.
BOLLIGER, M. \& L. WICK -1990- The pollen morphology of Odontites (Scrophulariaceae) and its taxonomic significance. Pl. Syst. Evol. 173: 159-178.

ERDTMAN, G. -1952-Pollen morphology and plant taxonomy - Angiosperms. Almqvist \& Wiksell, Stockholm.

ERDTMAN, G. -1960- The acetolysis method. A revised description. Sven. Bot. Tidskr. 39:561564.

JONES, G.D., V.M. Jr. BRYANT, M.H. LIEUX \& P.D. LINGREN -1995- Pollen of the Southeastern United States: with emphasis on melissopalynology and entomopalynology. AASP Contrib. Ser. 30: 1-76.

KHAN, H.A. -1968-Contributions to the pollen mophology of the Euphorbiaceae. J. Palynol. 4: 21-35.

LOBREAU-CALLEN, D. -1972- Pollen des Icacinaceae I. Atlas. Pollen Spores 14(4): 345388.

LOBREAU-CALLEN, D. \& S.K. SRIVASTAVA 1974- Paleocene ocurrence of Platea (Icacinaceae) Pollen in USA. Pollen Spores 16(3): 411-423.

MARTINSSON, K. -1993- The pollen of Swedish Callitriche (Callitrichaceae) - trends towards submergence. Grana 32:198-209.

MINKIN, J.P. \& W.H. ESHBAUGH -1989- Pollen morphology of the Orobanchaceae and rhinanthoid Scrophulariaceae. Grana 28: 1-18.

MULLER, J. -1973- Pollen morphology of Barringtonia calyptrocalyx K. Sch. (Lecitidaceae). Grana 13: 29-44.

PUNT, W., S. BLACKMORE, S. NILSSON, A. LE THOMAS -1994- Glossary of pollen and spore terminology. LPP Foundation, Utrecht.

ROSELLO, E.F. \& T.S. MELHEM -1998Palinotaxonomia de espécies brasileiras de Thymelaeaceae Juss. Bol. Bot. Univ. São Paulo 17: 1-24.

SALGADO-LABOURIAU, M.L. - 1973Contribuição à Palinologia dos Cerrados. Academia Brasileira de Ciências, Rio de Janeiro.

SALGADO-LABOURIAU, M.L., P.E. VANZOLINI \& T.S. MELHEM -1965- Variation of polar axes and equatorial diameters in pollen grains of two species of Cassia. Grana palynol. 6: 166-176. 
SANTOS, F.A.R. -1997- Scrophulariaceae do Brasil: morfologia polínica e suas aplicações taxonômicas. Tese de doutorado - Instituto de Biociências da Universidade de São Paulo. São Paulo.

SOUZA, V.C. -1996-Levantamento das espécies de Scrophulariaceae nativas do Brasil. Tese de doutorado - Instituto de Biociências da Universidade de São Paulo. São Paulo.
Aceptado para su publicación en julio de 2000

Endereço dos autores. F.A.R. Santos: Universidade Estadual de Feira de Santana, Departamento de Ciências Biológicas, Av. Universitária s/n, 44031460, Feira de Santana - BA, Brasil. E-mail: fasantos@uefs.br.T.S. Melhem: Instituto de Botânica, Seção de Dicotiledôneas, Caixa Postal 4005, 01061-970, São Paulo - SP, Brasil. E-mail: melhem@uol.com.br. 